A N N A L E S Annales de Bretagne et des Pays de l'Ouest

\title{
Le Bouclier de Neptune
}

La politique de défense des bases françaises en Méditerranée (1912-1931)

Jean-Baptiste Bruneau

\section{OpenEdition}

\section{Journals}

Édition électronique

URL : http://journals.openedition.org/abpo/3348

DOI : $10.4000 / a b p o .3348$

ISBN : 978-2-7535-5185-5

ISSN : 2108-6443

Éditeur

Presses universitaires de Rennes

\section{Édition imprimée}

Date de publication : 28 juillet 2016

Pagination : 199-200

ISBN : 978-2-7535-5183-1

ISSN : $0399-0826$

Référence électronique

Jean-Baptiste Bruneau, «Le Bouclier de Neptune », Annales de Bretagne et des Pays de l'Ouest [En ligne], 123-2 | 2016, mis en ligne le 28 juin 2016, consulté le 23 septembre 2020. URL : http:// journals.openedition.org/abpo/3348; DOl : https://doi.org/10.4000/abpo.3348 
viennent d'autres milieux que maritimes, et que finalement le clivage social est plus fort que le clivage d'appartenance ou non au monde des gens de mer.

Ce livre d'Alain Cabantous et Gilbert Buti fait donc la synthèse des deux orientations, culturelle d'une part, économique et sociale de l'autre, et réunit toutes les familles de marins, notamment les capitaines et le grand corps, puisqu'ils sont aussi " des hommes qui exercent le métier de marin ". Finalement, cet excellent livre aurait pu venir plus tôt. En 2007, lors du premier congrès du GIS d'Histoire maritime, Jacques Péret et Gilbert Buti avaient, dans cet esprit, présenté une excellente contribution sur l'évolution de la recherche concernant " Les gens de mer et sociétés littorales en France à l'époque moderne " (Revue maritime, n 10-11, 2010, p. 135-159). En effet, depuis, la recherche s'est déplacée vers d'autres centres d'intérêt dans une perspective plus internationale et interdisciplinaire, dans le cadre du GIS d'Histoire maritime devenu Histoire et sciences de la mer conventionné avec le CNRS. Ainsi, la recherche sur les gens de mer s'est-elle orientée vers le marché international de la main-d'œuvre maritime, les contrats d'engagement et les rémunérations, aussi bien en Méditerranée qu'en Atlantique, dans l'Océan Indien et la mer de Chine. Souhaitons que la question Etre marin? soit dans l'avenir abordée sur le temps plus long et dans une perspective mondiale.

Gérard LE BOUËDEC

SAFrRoy, Frédéric, Le Bouclier de Neptune. La politique de défense des bases françaises en Méditerranée (1912-1931), Rennes, PUR, coll. « Histoire », 2015, 391 p.

Avec la publication de la thèse de Frédéric Saffroy sur les fortifications côtières françaises de la première moitié du $\mathrm{Xx}^{\mathrm{e}}$ siècle se voit comblé un vide historiographique. Si les ouvrages et la pensée stratégique du " système Séré de Rivière ", tous comme ceux de la " ligne Maginot ", ont fait l'objet de nombreux travaux, la question du dispositif mis en place entre le début du siècle et la Seconde Guerre mondiale restait en souffrance et c'est un des grands mérites de cet ouvrage d'y répondre de manière étayée.

Si le sentiment de l'opinion publique au lendemain de la Première Guerre mondiale est d'avoir assisté à la faillite de la doctrine navale traditionnelle, qui prévoyait l'engagement des gros bâtiments de surface dans une très classique guerre d'escadre, les enseignements du conflit sur mer n'en sont pas moins considérables. En effet, l'arme sous-marine a montré toute l'efficacité de sa doctrine d'emploi, révélant par là même toute la dangerosité des atterrages qui nécessitent dès lors une protection renforcée. Si la défense des côtes n'est évidemment pas délaissée par la marine française à la veille du conflit, alors qu'elle se voit confier la responsabilité du bassin méditerranéen dans le cadre de la répartition entre les flottes alliées des théâtres d'opération, elle est rapidement mise à mal par les besoins de l'Armée qui, sur le front de l'Ouest, rapatrie tous les renforts en hommes et en artillerie lourde répartis sur le littoral. En réalité, à la veille du premier conflit mondial, malgré les réformes entreprises au début des années 1890, puis à nouveau en 1904 et 1913, l'Armée refuse de prendre réellement en compte les besoins spécifiques de la Marine, révélant une analyse antagoniste des besoins; ainsi, à la notion d'inviolabilité des frontières, posée comme un principe absolu par l'Armée, répond la protection des bases et arsenaux pour la Marine qui se soucie peu de la seule maîtrise territoriale. La guerre venue, la contradiction éclate au détriment de la Marine. Dépouillée de son artillerie lourde, elle n'a pourtant de cesse de reprendre la main sur la défense des 
côtes qu'elle finit, en septembre 1917, par se voir enfin transférer, prélude à la récupération, en janvier 1918, de l'ensemble des fortifications et du matériel.

Au lendemain de la victoire, malgré la déception qui entoure l'action de la marine française au cours du conflit, la question de sa pérennité ne saurait être sérieusement envisagée. En revanche, celle de son format reste d'autant plus en suspens que la guerre a ruiné les espoirs de " bataille décisive " envisagée dans la continuité des thèses mahaniennes, ruinant toutes les conceptions stratégiques, conditions du matériel, qui s'étaient imposées de manière hégémonique avant-guerre. Une marine oui, mais pour quoi faire? C'est pour répondre à cette question, et présenter au Parlement un format cohérent, qu'est élaboré, à partir de 1923, par le Conseil supérieur de la Marine un statut naval, reposant sur les impératifs dit des " besoins absolus ", qui comprend quatre volets : le format de la flotte, l'approvisionnement en combustibles liquides, l'aéronautique maritime et l'organisation de la défense des frontières maritimes. Avec ce statut, la politique navale de la France dispose d'un fil rouge qui va s'imposer, non sans limites, au cours de l'Entre-deux-guerres.

En ce qui concerne le dernier volet de ce statut, une des questions pendantes est celle de définir la forme qu'elle doit prendre : flottante ou terrestre, fixe ou mobile, directe ou indirecte. Dans cette recherche de la défense idéale, les stratèges et historiens, tel l'amiral Daveluy, fort de son expérience de commandant du front de mer de Toulon, mettent en avant la nécessité d'une réorganisation de la défense des côtes qui doit être concentrée autour des bases et arsenaux, d'un renforcement de l'artillerie, et de la mise en place d'une défense anti-aérienne dont le conflit a montré l'importance. Si les deux premières mesures sont rapidement entérinées, notamment grâce aux stipulations de la conférence de désarmement de Washington qui permettent le transfert des canons des navires désarmés vers les batteries côtières, la question de la menace aérienne reste en suspens. Outre les pesanteurs, liées notamment à la question de l'attribution des forces aériennes qui se pose à toutes les grandes nations qui sortent du conflit sans avoir réglé la question de cette arme nouvelle, et le conservatisme de l'institution, les difficultés financières interdisent toute prise en compte sérieuse de cette menace.

Loin d'être cantonnés aux cercles militaires, les débats entourant les différentes options envisagées pour cette nouvelle organisation de la défense des côtes témoignent, sous la plume ici particulièrement claire de Frédéric Saffroy, de la parfaite maîtrise des enjeux stratégiques par les parlementaires de la commission de la Marine, démentant une nouvelle fois la légende installée par l'amiral Darlan, et depuis assez complaisamment entretenue, de l'ignorance de la Chambre pour la chose militaire. Outre le rappel de la place cardinale tenue par le Parlement, dont Frédéric Saffroy souligne qu'il " a également orienté la Marine quand celle-ci n'était pas suffisamment prête ", cette étude permet aussi de réévaluer la place, dans cette refondation des structures de la marine de l'Entre-deux-guerres, d'un ministre écrasé par la figure tutélaire de Georges Leygues, Flaminius Raiberti dont l'impulsion et la constance dans l'implication, rue Royale mais aussi au Sénat, ont été décisives.

Jean-Baptiste BRUNEAU 